\title{
EL IMPACTO DEL USO DEL CORREO ELECTRÓNICO EN EL PROFESORADO DE LAS UNIVERSIDADES PÚBLICAS MADRILEÑAS
}

\author{
Irene Albarrán Lozano \\ Universidad Complutense de Madrid \\ Carmen de Pablos Heredero \\ Universidad Rey Juan Carlos \\ Antonio Montero Navarro \\ Universidad Rey Juan Carlos
}

\begin{abstract}
RESUMEN
El correo electrónico es uno de los medios de comunicación que se perfila como fundamental en las comunicaciones en los próximos años. El ámbito universitario no puede ser ajeno a esta realidad, por sus características propias, tanto en la faceta docente como investigadora.

Con el fin de comprobar la implantación real del correo electrónico en las universidades públicas madrileñas, hemos diseñado y realizado una encuesta que trata de recoger la opinión de los docentes en torno a su propio uso y conocimiento del correo electrónico y otros medios informáticos, así como su actitud hacia los mismos. Igualmente, nos hemos interesado por algunas cuestiones relevantes en torno al correo, como son su grado de satisfacción y las expectativas que genera.

Se ha contrastado empíricamente la existencia de diversas relaciones, destacando la influencia de las actitudes previas en los niveles de conocimiento de las herramientas, y la de dicho conocimiento en la posterior utilización de las mismas. Del mismo modo, hemos podido constatar la existencia de notables diferencias entre las diversas áreas de conocimiento, categorías profesionales y universidades.
\end{abstract}

\section{LAS COMUNICACIONES. EL CORREO ELECTRÓNICO}

\subsection{INTRODUCCIÓN}

La electrónica, la informática y las telecomunicaciones no son ya nuevas tecnologías, sino que se han ido desarrollando de forma progresiva en el últi- 
mo siglo y medio. Hasta hoy se han conseguido grandes capacidades de cálculo, de proceso y de almacenamiento de datos. Hoy las telecomunicaciones llegan a todas partes y permiten la transmisión de grandes cantidades de información, gracias a la extensión de las redes por cable y por ondas.

Las posibilidades actuales para tratar y transmitir la información permiten influir en los aspectos sociales que están relacionados con la comunicación. De la misma forma que la Revolución Industrial originó en los últimos doscientos años modificaciones en las estructuras productivas, se prevén cambios sociales derivados de un uso generalizado de las telecomunicaciones. Los cambios apuntan fundamentalmente a modificar las relaciones interpersonales, pasando de relaciones más lentas a otras más rápidas, de escasez a abundancia de información. La aparición, ya en nuestro siglo, de tecnologías telemáticas, especialmente en la década de los noventa, ha cambiado no solamente la forma en la que accedemos al conocimiento y cómo se produce el mismo, sino además las relaciones que establecemos a la hora de mantenernos comunicados.

Como señala Rheingold (1996), las comunicaciones mediadas por ordenadores (CMC) tienen el potencial de cambiar nuestras vidas desde varios niveles diferentes mutuamente influyentes. Como humanos, tenemos percepciones, personalidades y pensamientos que ya transmitíamos a través de otras tecnologías y que afectan a cómo utilizamos el medio. En un nivel de interacción de persona a persona, la tecnología permite posibilidades de comunicación multilaterales.

Así como otros medios previos permitieron disolver barreras sociales referentes al tiempo y espacio, el correo electrónico ayuda a disolver las fronteras de identidad. No hay que olvidar que la forma en la que las personas hacemos uso de las tecnologías afecta a nuestra conducta, pensamientos y a las relaciones que generamos.

Las nuevas facilidades para el tratamiento y transmisión de la información inciden de lleno en los aspectos sociales relacionados con la comunicación. La sociedad está formada por grupos interconectados y superpuestos que se caracterizan por desarrollar valores e intereses compartidos, normas de interacción, identidad y conciencia de grupo particular. Un uso adecuado de las tecnologías de información puede llegar a provocar cambios en el proceso de socialización de la estructura de grupos sociales.

\subsection{LAS TECNOLOGÍAS DE INFORMACIÓN Y COMUNICACIÓN. EL CORREO ELECTRÓNICO}

La implantación de tecnología de información en las organizaciones y las consecuencias estratégicas de la misma se han estudiado bajo diversas perspectivas. Porter (1985), Sethi y King (1994), Toraskar y Joglekar (1993) hacen referencias a casos concretos de obtención de ventajas por un uso apropiado de las tecnologías de información en los procesos de comunicación. Para McKeen y Smith (1993), el uso de la tecnología de información puede tener repercusio- 
nes no sólo en las relaciones entre organizaciones, sino también en las relaciones internas de las mismas. Brown (1995) menciona las inversiones en sistemas de información estratégicos como una forma a través de la que las organizaciones buscan ventajas competitivas.

Dos Santos (1993) mantiene que el impacto de las inversiones en tecnología de información en la organización no puede comprenderse de forma completa atendiendo únicamente al desempeño medido en altos niveles de creación de valor, puesto que no lleva a distinguir las aplicaciones y usos de la misma. Para entender el impacto de una determinada tecnología dentro de las organizaciones, parece apropiado examinar cómo se usa en los procesos en los que se está aplicando. Resulta útil identificar diferencias en cómo se percibe la tecnología de información por los usuarios en una organización como medio de buscar competitividad y cómo esta percepción se lleva a cabo en la realidad.

Las tecnologías de información están afectando a la forma de intercambio de información entre diferentes miembros de las organizaciones. Permiten, en muchos casos, sustituir el contacto físico. Numerosos autores han examinado de forma empírica el papel de los medios electrónicos en la comunicación (Eveland y Bikson, 1988; Finfholt y Sproull, 1990; Markus, 1994; Rice y Asociados, 1984; Fulk, 1993; Sproull y Kiesler, 1991; Trevino, Lengel y Daft, 1987).

Yates y Orlikowski $(1992 ; 1994)$ hablan de géneros de comunicación organizativa, como por ejemplo una memo, una reunión de comité o un resumen, en cuanto acciones tipificadas comunes y reconocidas de forma social como una manera con un propósito y características comunes determinados. El propósito de comunicación de un género no es un motivo individual privado para comunicar, sino un propósito construido y reconocido por la comunidad y usado para ciertas situaciones. Por ejemplo, el propósito socialmente reconocido de una reunión de un comité es discutir, tomar decisiones, delegar o implantar acciones relativas a sus competencias.

Ricoma (1996) habla de dos tipos de tecnologías disponibles para la comunicación en las organizaciones: tecnologías de pantalla ${ }^{1}$ o tecnologías de voz y datos $^{2}$, donde podríamos incluir el fax, el correo electrónico y las tecnologías de voice and mail.

En los últimos quince años, el uso de ordenadores personales ha popularizado un número considerable de servicios de información, incluyendo el correo electrónico. El e-mail es una forma de intercambio de información en la que se mandan mensajes de un ordenador personal o terminal a otro vía módem y sistemas de telecomunicaciones.

El uso del correo comenzó con ARPAnet (red precursora de Internet) en 1969 y 1970 en los Estados Unidos, se extendió de forma gradual con el uso de los mainframes y miniordenadores basados en redes locales en los setenta, y tuvo un rápido crecimiento con el uso de Internet en la década de los ochenta.

1 Del inglés face to screen.

2 Voice and data. 
El correo electrónico en sus inicios se plantea como un medio de intercambio de información para grupos pequeños y selectos. Actualmente, su uso se ha extendido a millones de usuarios por todo el mundo. El correo electrónico es el servicio más utilizado de los que existen hoy en Internet. Desde el año 1970 se ha empleado como herramienta de comunicación para relaciones académicas y personales. Por el año 1990, la popularidad y ubicuidad del correo sobre el resto de los medios de comunicación tradicionales ha permitido que se reconozca como medio estándar de comunicación.

El correo electrónico permite el envío de mensajes por medios informáticos. Los mensajes se almacenan en un buzón personal. Cuando cada usuario consulta su correspondencia puede visualizar, almacenar o reenviar mensajes recibidos. Los mensajes enviados pueden estar en cualquier tipo de formato, texto, gráficos, imágenes, sonido, etc.

El e-mail es un medio electrónico que permite la instantaneidad de comunicación entre receptor y emisor. Sáenz Vacas hace referencia al correo electrónico bajo las siglas EAUDI, tratando de destacar las características típicas que hacen a este medio idóneo para ciertos tipos de comunicación en las organizaciones (electrónico, asíncrono, ubicuo, digital e informático).

- Electrónico: utiliza medios electrónicos de gestión y transporte.

- Asíncrono: no necesita sincronía en envío y recepción.

- Ubicuo: permite su acceso en diferentes lugares.

- Digital: utiliza información digitalizada.

- Informático: está en relación con las tecnologías de la información.

A continuación pasamos a citar algunas de las principales ventajas e inconvenientes de este medio de comunicación, especialmente en lo referente al entorno universitario.

\section{TABLA 1}

Ventajas de los diversos modos de comunicación

Rapidez y fiabilidad en la recepción y envío de Incrementa el contacto personal mensajes

No requiere simultaneidad del remitente y el Permite una mejor transmisión de los mensajes receptor complejos

Facilidad de archivo, reenvío e integración

Mejora la consistencia de los mensajes

Bajo coste

Incrementa la motivación y el ánimo

Facilita la comprensión en algunos casos 


\section{OBJETIVOS DEL ESTUDIO}

En un entorno como el universitario, donde se da una necesidad importante de establecer comunicaciones, con objetivos fundamentales de investigación entre personas y equipos de trabajo, el uso del correo electrónico nos parece fundamental. En el ámbito universitario español no existe generalmente una política específica que establezca pautas sobre uso del correo electrónico. En el mercado norteamericano se han tomado ya iniciativas a este respecto, tratando de desarrollar políticas de utilización de esta herramienta, buscando una consistencia con misión y objetivos universitarios (Anderson et al., 1996).

Si bien se han realizado diversos estudios sobre la utilización del correo electrónico en empresas privadas, es menos habitual encontrar trabajos basados en organismos relacionados con las Administraciones públicas. Consideramos interesante conocer algunos datos relativos a la utilización del correo electrónico por parte de los docentes, por cuanto se trata de un cuerpo profesional con una clara influencia en la formación tecnológica de los futuros trabajadores.

Nuestro estudio, llevado a cabo entre los docentes de las diversas universidades públicas de la Comunidad Autónoma de Madrid, pretende fundamentalmente:

- Realizar una breve descripción de la actitud, utilización, grado de satisfacción y expectativas de los medios informáticos, en especial el correo electrónico, dentro del ámbito de estudio.

- Comprobar la existencia de relaciones entre algunas de las variables del estudio. Concretamente, hemos estudiado la influencia de la actitud hacia los diversos medios con el conocimiento de los mismos y su utilización.

- Mostrar algunas de las principales diferencias y similitudes existentes entre las diversas universidades objeto del estudio.

\section{METODOLOGÍA}

Con el fin de conocer los principales parámetros que caracterizan la utilización del correo electrónico en la universidad, se envió un cuestionario de respuesta anónima a los docentes de las universidades públicas madrileñas ${ }^{3}$ durante los meses de noviembre y diciembre de 1998, y a principios del mes de enero de 1999. En estos centros trabajan un total de 13.648 docentes e investigadores, según las últimas cifras disponibles, que datan del principio del curso académico 1998-1999.

${ }^{3}$ Universidad Autónoma de Madrid, Universidad Complutense de Madrid, Universidad de Alcalá, Universidad Politécnica de Madrid, Universidad Carlos III y Universidad Rey Juan Carlos. 
El cuestionario consta de cinco bloques, compuestos por un total de 23 preguntas. Estos bloques se refieren a diversos aspectos:

- Datos personales, en el que se pretende identificar las principales características de los encuestados, con el fin de poder delimitar con posterioridad algunos perfiles de usuarios del correo.

- Actitud previa, en el que se ha tratado de conocer y valorar cuál es la actitud mostrada por los encuestados ante los medios informáticos en general, y hacia el correo electrónico en particular.

- Formación, en el que se ha preguntado sobre el nivel de conocimiento de algunos de los medios electrónicos que consideramos más importantes a la hora de desarrollar una labor docente e investigadora, así como la utilización de dichos medios. Asimismo, se intenta conocer de qué modo se ha llegado al actual grado de conocimiento del correo electrónico.

- Utilización del correo electrónico, en el que se trata de valorar cuestiones dispares relativas al nivel de uso del correo, el contenido de los mensajes o las recomendaciones acerca de su utilización.

- Satisfacción y expectativas, parte en la que se trata de conocer cuál es el grado de satisfacción que presentan los diversos usuarios del correo, la valoración de este medio de comunicación y el planteamiento de algunos de sus principales problemas.

A continuación presentamos la tabla que recoge las distintas preguntas que han compuesto el cuestionario, y las variables con las que han sido relacionadas para su tratamiento estadístico. 


\section{TABLA 2}

\section{Cuestionario}

$$
\text { Preguntas Tipo }
$$

\section{Bloque 1: Datos personales}

Edad Cualitativa. Categórica (de 1 a 5)

Universidad Cualitativa. Categórica (de 1 a 6 )

Área de conocimiento Cualitativa. Categórica (de 1 a 5)

Años trabajando en la universidad Cuantitativa. Discreta

Años desempeñando el trabajo actual Cuantitativa. Discreta

Categoría profesional Cualitativa. Categórica (de 1 a 7 )

\section{Bloque 2: Actitud previa}

Actitud hacia los medios informáticos

Cualitativa. Categórica (de 1 a 5)

Actitud hacia el correo

\section{Bloque 3: Formación}

Nivel de conocimiento sobre procesadores de textos ........... Cualitativa. Categórica (de 0 a 5)

Nivel de conocimiento sobre hojas de cálculo Cualitativa. Categórica (de 0 a 5 )

Nivel de conocimiento sobre Internet Cualitativa. Categórica (de 0 a 5)

Nivel de conocimiento sobre correo eléctrico Cualitativa. Categórica (de 0 a 5)

Nivel de conocimiento sobre bases de datos digitales Cualitativa. Categórica (de 0 a 5 )

Nivel de conocimiento sobre paquetes matemático-estadísticos .... Cualitativa. Categórica (de 0 a 5)

Nivel de uso sobre procesadores de textos Cualitativa. Categórica (de 0 a 5 )

Nivel de uso sobre hojas de cálculo Cualitativa. Categórica (de 0 a 5 )

Nivel de uso sobre Internet Cualitativa. Categórica (de 0 a 5)

Nivel de uso sobre correo electrónico Cualitativa. Categórica (de 0 a 5)

Nivel de uso sobre bases de datos digitales Cualitativa. Categórica (de 0 a 5)

Nivel de uso sobre paquetes matemático-estadísticos Cualitativa. Categórica (de 0 a 5)

Modo en el que ha llegado a su actual grado de conocimiento del correo

\section{Bloque 4: Utilización del corre electrónico}

Tiempo que utiliza diariamente el correo electrónico

Cuantitativa. Discreta

Número de mensajes (e-mails) que envía diariamente Cuantitativa. Discreta

Porcentaje de mensajes cuyo contenido es estrictamente laboral ... Cuantitativa. Discreta

Porcentaje de mensajes cuyo contenido no es laboral Cuantitativa. Discreta

Porcentaje de mensajes que no llegan a su destino Cuantitativa. Discreta

Posición ante el empleo del sentido del humor en los mensajes .... Cualitativa. Categórica (de 1 a 5)

\section{Bloque 5: Satisfacción y expectativas}

Ventajas del uso del correo electrónico Cualitativa. Abierta

Principales problemas del uso del correo electrónico Cualitativa. Abierta ¿Sabía la existencia de empresas que controlan los mensajes? Cualitativa. Dicotómica ¿Considera adecuada o justificada esta actuación? Explíquelo Cualitativa. Dicotómica. Abierta ¿Existen en su trabajo normativas relativas al uso del correo? Cualitativa. Dicotómica

¿Existen medidas de seguridad específicas? En caso afirmativo, indique cuáles Cualitativa. Dicotómica. Abierta ¿Considera al correo electrónico un medio seguro de comunicación? Cualitativa. Dicotómica 


\section{RESULTADOS}

\subsection{ANÁLISIS DESCRIPTIVO}

\subsubsection{Datos generales}

El plazo de recepción de encuestas, tras alguna ampliación, se cerró de manera definitiva el día 1 de marzo. En esta fecha se habían recibido 910 respuestas, aun cuando existen valores perdidos en algunas de las preguntas realizadas. De éstas, la mayoría corresponden a profesores de la Universidad Complutense de Madrid (273, un 30,7\%), seguida de la Universidad Autónoma de Madrid (166), la Universidad Politécnica de Madrid (163), la Universidad de Alcalá de Henares (126), la Universidad Carlos III (118) y la Universidad Rey Juan Carlos (43)

Como podemos observar, la mayor parte de las respuestas corresponden a profesores de la Universidad Complutense de Madrid. Esto se debe, en parte, a la mayor cantidad de docentes que trabajan en esta Universidad, que es actualmente una de las de mayor tamaño de España. A continuación, podemos observar igualmente el porcentaje de respuestas en cada una de las universidades en relación a su personal docente. El nivel de respuestas obtenido fue superior al 6\%, alcanzándose los mejores índices en las universidades nuevas (UC3M y URJC), junto a la de Alcalá de Henares.

\section{TABLA 3}

Porcentajes de respuestas por universidades

\begin{tabular}{lrrrrrrr}
\hline & $\begin{array}{c}\text { Complu- } \\
\text { tense }\end{array}$ & $\begin{array}{r}\text { Autó- } \\
\text { noma }\end{array}$ & $\begin{array}{c}\text { Poli- } \\
\text { técnica }\end{array}$ & Alcalá & Carlos III & $\begin{array}{c}\text { Rey Carlos } \\
\text { Carlon }\end{array}$ & TOTAL \\
\hline Profesores ......... & 5.863 & 2.034 & 3.182 & 1.149 & 815 & 391 & 13.434 \\
Respuestas ........ & 273 & 166 & 163 & 126 & 118 & 43 & 889 \\
Porcentaje .......... & 4,8 & 8,4 & 5,2 & 11,2 & 14,8 & 11,3 & 6,8 \\
\hline
\end{tabular}

Por áreas de conocimiento, predominan los docentes de Ciencias Sociales (262), Ingeniería (234) y Ciencias Experimentales (210), frente a los profesores de Ciencias de la Salud (100) o los de Humanidades (103).

Por último, en la tabla 4 podemos comprobar los índices de respuesta por categorías profesionales. Al margen del elevado índice de respuesta de la categoría genérica "otros», que recoge a los profesores eméritos, visitantes y otras variedades contractuales, cabe destacar a los profesores ayudantes, que también superan el $10 \%$ de respuestas. 


\section{TABLA 4}

Porcentajes de respuestas por categorias profesionales

\begin{tabular}{lrrrrrrr}
\hline & $\begin{array}{c}\text { Catedrá- } \\
\text { ticos } U\end{array}$ & $\begin{array}{c}\text { Titulares } \\
\text { Cicatedrá- }\end{array}$ & $\begin{array}{r}\text { Citulares } \\
\text { ticos EU }\end{array}$ & \multicolumn{1}{c}{ EU } & Asociados & Ayudantes & Otros \\
\hline Profesores ......... & 1.505 & 4.411 & 225 & 1.970 & 4.538 & 785 & 116 \\
Respuestas ....... & 129 & 347 & 12 & 97 & 162 & 100 & 44 \\
Porcentaje ......... & 8,57 & 7,87 & 5,33 & 4,92 & 3,57 & 12,74 & 37,93 \\
\hline
\end{tabular}

Podríamos afirmar, en función a las frecuencias de las respuestas obtenidas, que el profesor que ha respondido al cuestionario es, en promedio, un profesor titular de universidad, cuya experiencia en su universidad difiere bastante en función de cuál sea ésta (nueva o más antigua), que lleva aproximadamente nueve años en su actual puesto u otro similar, y que tiene en torno a los 40 años de edad (con fuerte diferencia en la URJC).

\subsubsection{Actitud previa frente al correo electrónico y los medios informáticos}

La actitud hacia los medios informáticos y el correo electrónico es francamente positiva en casi todos los casos. Los valores generales señalan una actitud positiva o muy positiva hacia el correo en un $96 \%$ de las contestaciones, reduciéndose ligeramente (92\%) la cifra si nos referimos a la actitud hacia los medios informáticos.

La tabla 5 resume las ligeras diferencias entre universidades. Todas ellas presentan una actitud hacia el correo completamente positiva en la mayoría de las contestaciones, pero no ocurre lo mismo con la actitud hacia los medios informáticos.

\section{TABLA 5}

Actitud hacia los medios informáticos y el correo electrónico por universidades (en porcentajes)

\begin{tabular}{|c|c|c|c|c|c|c|}
\hline & $U C M$ & $U A M$ & $U P M$ & $U A H$ & $U C 3 M$ & URJC \\
\hline Actitud correo .... & «++» $(62)$ & «t+» $(68,1)$ & «++» $(66,4)$ & «++» $(62,7)$ & «++» $(72,0)$ & «++» $(62,8)$ \\
\hline Actitud medios ... & «+» (49) & «+» $(48,2)$ & «++» $(57,1)$ & «++» $(49,2)$ & «++» $(53,4)$ & «+» $(51,2)$ \\
\hline
\end{tabular}


La actitud hacia los medios informáticos es positiva («+») en todas las categorías profesionales, salvo en los profesores asociados, que muestran una actitud completamente positiva («++»). En cuanto a áreas de conocimiento, nuevamente la mayoría son actitudes positivas, salvo en Ingenierías (también completamente positiva). La actitud predominante hacia el correo es, sin embargo, completamente positiva, salvo en el área de Humanidades y en la categoría de Catedrático de Escuela (simplemente positivas).

\subsubsection{Conocimiento y uso de las herramientas informáticas}

En la tabla 6 podemos observar los datos generales relativos a conocimientos y utilización del correo electrónico. Se han clasificado nuevamente las respuestas en tres niveles distintos, bajo, medio y alto, respectivamente (1, 2 y 3$)$

Deducimos la existencia de dos grupos de herramientas informáticas, que podríamos denominar sencillas y complejas. Así, los encuestados muestran por lo general altos niveles de conocimiento y utilización del correo electrónico, procesadores de texto e Internet, mientras que los niveles relativos a bases de datos digitales, hojas de cálculo y paquetes matemático-estadísticos son sensiblemente inferiores. Estos dos grupos se caracterizan por distintos comportamientos: mientras que en las que hemos denominado sencillas el uso va por delante del conocimiento de forma clara, las complejas son aprendidas generalmente de cara a su utilización, de modo que conocimiento y uso van parejos.

\section{TABLA 6}

Conocimiento y uso del correo electrónico

\begin{tabular}{|c|c|c|c|c|}
\hline & Media & $1(\%)$ & $2(\%)$ & $3(\%)$ \\
\hline \multicolumn{5}{|l|}{ Conocimiento } \\
\hline Hojas de cálculo ............................. & 2 & 30,2 & 42,8 & 27,0 \\
\hline Correo electrónico ......................... & 2,5 & 4,2 & 29,9 & 65,9 \\
\hline Bases de datos digitales .................. & 1,7 & 47,8 & 34,3 & 17,9 \\
\hline 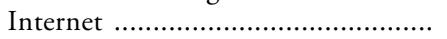 & 2,3 & 8,9 & 49,8 & 41,3 \\
\hline Paquetes estadísticos ........................ & 1,7 & 45,1 & 35,4 & 19,6 \\
\hline Procesador de textos .......................... & 2,7 & 5,0 & 26,8 & 72,7 \\
\hline \multicolumn{5}{|l|}{ Uso } \\
\hline Hojas de cálculo ............................ & 1,8 & 41,1 & 31,2 & 27,7 \\
\hline Correo electrónico ........................... & 2,7 & 5,2 & 16,6 & 78,2 \\
\hline Bases de datos digitales ................... & 1,6 & 55,3 & 27,9 & 16,9 \\
\hline 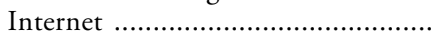 & 2,4 & 13,7 & 38,4 & 47,9 \\
\hline Paquetes estadísticos .......................... & 1,6 & 52,0 & 27,6 & 20,4 \\
\hline 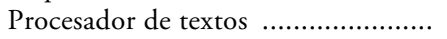 & 2,8 & 1,0 & 11,2 & 87,7 \\
\hline
\end{tabular}


En la tabla 7 recogemos los valores medios que se han obtenido para el conocimiento y uso de las herramientas informáticas en función del área de conocimiento.

\section{TABLA 7}

Conocimiento y uso de herramientas informáticas por áreas de conocimiento

\begin{tabular}{lccccc}
\hline & Humanidades & C. Sociales & Ingenierías & C. Experimentales & C. Salud \\
\cline { 2 - 6 } & & & & & \\
Conocimiento & 1,4 & 2 & 2,2 & 1,8 & 1,8 \\
Hojas de cálculo ...... & 2,4 & 2,5 & 2,7 & 2,5 & 2,4 \\
Correo .................. & 1,5 & 1,7 & 1,8 & 1,5 & 1,6 \\
Bases de datos ......... & 2,1 & 2,3 & 2,5 & 2,2 & 2,1 \\
Internet ............... & 1,1 & 1,8 & 1,9 & 1,7 & 1,5 \\
Paquetes estadísticos ... & 2,7 & 2,7 & 2,8 & 2,6 & 2,5 \\
Procesador textos .... & & & & & \\
& & & & & \\
Uso & 1,3 & 1,9 & 2,1 & 1,6 & 1,8 \\
Hojas de cálculo ...... & 2,7 & 2,6 & 2,8 & 2,7 & 2,5 \\
Correo ................... & 1,5 & 1,6 & 1,5 & 1,5 & 1,6 \\
Bases de datos ......... & 2 & 2,3 & 2,5 & 2,2 & 2,2 \\
Internet ................ & 1,1 & 1,8 & 1,7 & 1,6 & 1,5 \\
Paquetes estadísticos ... & 2,8 & 2,8 & 2,8 & 2,8 & 2,7 \\
Procesador textos .... & 2,8 & & & & \\
\hline
\end{tabular}

Los profesores de Ingeniería son los que afirman tener mayores conocimientos de todas las herramientas informáticas, y los de Humanidades son, en cambio, los que muestran menores niveles. Esta diferencia se repite para el uso de dichas herramientas. La similitud en las cifras obtenidas para el caso del conocimiento de procesadores de textos indica de forma clara que esta herramienta es igualmente muy conocida y utilizada para las diferentes áreas de conocimiento, independientemente de su especialización.

Esta diferencia se reproduce entre universidades, ya que prácticamente todos los profesores de la Universidad Politécnica pertenecen al área de Ingeniería, de forma que ésta presenta resultados de conocimiento y uso más elevados para todos los medios informáticos. Por categorías profesionales, podemos destacar que quienes tienen un mayor conocimiento de estas herramientas son los Ayudantes (muestran medias superiores a 2). En general, todas las categorías presentan un alto grado de conocimiento de los procesadores de textos y correo electrónico. Los que menos conocen y utilizan tanto el correo como las bases de datos son los Catedráticos de Escuela. Curiosamente, los Titulares de Universidad son los que menos utilizan las bases de datos e Internet en general. 
El modo más habitual de adquirir su conocimiento sobre el correo electrónico es, con gran diferencia en todas las universidades, autodidacta. En la Rey Juan Carlos, la Politécnica y Alcalá de Henares, el porcentaje es superior al $50 \%$. Existen, además, numerosas respuestas que incluyen una combinación de esta opción con alguna de las otras, esto es, con una breve explicación de amigos o conocidos, con un curso de la Universidad o con otro tipo de curso.

\subsubsection{Utilización del correo electrónico}

En este bloque, buscamos conocer algunos detalles sobre la utilización del correo electrónico por parte de los docentes. El tiempo medio de utilización del correo electrónico es de 32 minutos (el 27,7\% de las respuestas lo utilizan media hora al día). Sin embargo, hay que reseñar que existe una fuerte dispersión (desviación típica de 35 minutos), y la presencia de un colectivo superior al $15,5 \%$ que utiliza diariamente el correo electrónico durante una hora o más tiempo. Por universidades, las respuestas oscilan entre valores medios de 40 minutos de los profesores de la Universidad Politécnica y 23 minutos de los profesores de la Universidad Rey Juan Carlos.

En cuanto al número de mensajes que se envían, podemos comprobar que en media son cuatro, aunque de nuevo existe una importante dispersión $(7,8$ de desviación típica). Un $24 \%$ de los profesores que han respondido mandan dos mensajes diarios y el $22 \%$ remiten uno. Curiosamente, hay una persona que admite enviar diariamente 98 mensajes. Nuevamente es la Universidad Politécnica la que destaca sobre las demás (6 mensajes diarios), y por áreas, Ingeniería.

\section{TABLA 8}

Utilización del correo electrónico

\begin{tabular}{|c|c|c|c|c|c|c|c|}
\hline & $U C M$ & $U A M$ & $U P M$ & $U A H$ & $U C 3 M$ & URJC & Media \\
\hline $\begin{array}{l}\text { Tiempo de utilización diario } \\
\text { (minutos) }\end{array}$ & 27 & 35 & 40 & 27 & 39 & 24 & 32 \\
\hline $\begin{array}{l}\text { Número de mensajes enviados } \\
\text { Mensajes estrictamente laborales }\end{array}$ & 3,4 & 4 & 6 & 3 & 4 & 3 & 4 \\
\hline $\begin{array}{l}\text { Mensajes estrictamente laborales } \\
\text { (todos) }(\%)\end{array}$ & 38,00 & 37,80 & 36,10 & 41,30 & 26,70 & 27,50 & 36,20 \\
\hline $\begin{array}{l}\text { Mensajes no laborales (ninguno) } \\
\text { (\%) }\end{array}$ & 38,70 & 38,60 & 39,60 & 30,40 & 30,40 & 33,30 & 39,30 \\
\hline $\begin{array}{l}\text { Todos los mensajes llegan a su } \\
\text { destino (\%) }\end{array}$ & 48,40 & 57,20 & 57 & 42,20 & 53,20 & 34,30 & 50 \\
\hline No pertenencia a una lista (\%)... & 50 & 27,50 & 41,40 & 32,80 & 24,10 & 64,20 & 39 \\
\hline $\begin{array}{l}\text { Sentido del humor (elección vo- } \\
\text { luntaria) }(\%)\end{array}$ & 52 & 56,50 & 52,20 & 55,90 & 61,70 & 50 & 50,50 \\
\hline
\end{tabular}


Con relación al porcentaje de mensajes con carácter plenamente laboral, más de un tercio de los profesores afirman que todos lo son, mientras que son dos tercios los que aseguran que más de un $75 \%$ de sus mensajes son de carácter plenamente laboral. Simultáneamente, casi cuatro de cada diez profesores jamás envían mensajes no laborales, y sólo un $25 \%$ envía una cantidad de mensajes no laborales superior al $25 \%$. Tales cifras son sensiblemente mejores que las obtenidas en prácticamente todos los estudios realizados en empresas, por lo que podemos pensar que, a tenor de nuestras respuestas, los profesores universitarios somos más conscientes de la necesidad de aprovechar bien los recursos que los profesionales que trabajan en todo tipo de empresas. En cualquier caso, y al margen de esta consideración, hemos podido contrastar que existe una independencia absoluta entre la variable "uso del correo" y el porcentaje de mensajes estrictamente laborales (esto es, la primera no condiciona a la segunda). La Universidad Rey Juan Carlos conjuga dos facetas curiosas, como son el mayor número de mensajes estrictamente laborales y la mayor cantidad de mensajes de carácter no laboral. Por categorías, el mayor porcentaje de mensajes estrictamente laborales corresponde a los Catedráticos de Facultad, mientras que los Ayudantes y Otros son los que menor porcentaje presentan en este sentido.

Aproximadamente la mitad de los docentes afirma que todos sus mensajes llegan a su destino, y la mayor parte del resto de respuestas indican que la cantidad de mensajes perdidos es muy escasa. El menor porcentaje de mensajes que se pierden corresponde a Ingeniería (a pesar del mayor número de mensajes de promedio), frente a Humanidades, lo cual puede tener una relación con la formación tecnológica de los docentes.

La tabla 9 muestra el porcentaje de respuestas que indican la opinión de los docentes sobre si se debe utilizar un cierto sentido del humor en los mensajes que se envían a través del correo electrónico.

\section{TABLA 9}

Sentido del humor en los mensajes de correo electrónico

\begin{tabular}{|c|c|}
\hline Aspecto considerado & Porcentaje de respuestas \\
\hline Es una pérdida de tiempo y recursos. & 11 \\
\hline Se malinterpreta fácilmente & 6,5 \\
\hline 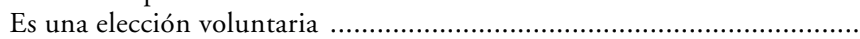 & 55,6 \\
\hline Es bueno & 19,4 \\
\hline Es adecuado, incluso en los mensajes profesionales & 7,6 \\
\hline
\end{tabular}

Dejando a un lado las respuestas que afirman que se trata de una opinión, parece que existe una mayor cantidad de opiniones favorables que contrarias. 
La respuesta a esta pregunta es bastante uniforme por universidades y áreas de conocimiento, aunque existe una ligera divergencia por categorías. Podemos destacar que los Profesores Titulares de Escuela Universitaria perciben mayoritariamente que el sentido del humor es una pérdida de tiempo y recursos.

\subsubsection{Satisfacción y expectativas}

En el último bloque del cuestionario hemos tratado de indagar sobre ventajas y problemas propios del uso del correo electrónico en este ámbito, así como cerciorarnos de la percepción de los encuestados sobre la problemática de la seguridad en las redes y conocer otras opiniones.

\section{TABLA 10}

Satisfacción y expectativas sobre el correo electrónico (en porcentajes)

\begin{tabular}{|c|c|c|c|c|c|c|c|}
\hline & $U C M$ & $U A M$ & $U P M$ & $U A H$ & $U C 3 M$ & URJC & Media \\
\hline Existencia de normas sobre correo ............. & 6,20 & 12,90 & 11,70 & 6,70 & 33,30 & 0 & 11,90 \\
\hline Conoc & 16,50 & 35,40 & 44,90 & 10,50 & 41,10 & 29,30 & 37,80 \\
\hline Cono & 9,40 & 19,80 & 20,30 & 20 & 51,60 & 26,30 & 27,70 \\
\hline Consideran correo medio seguro ................ & 49 & 54,20 & 49,40 & 54,20 & 67,30 & 46,30 & 53,40 \\
\hline
\end{tabular}

El correo electrónico es, generalmente, considerado un medio seguro de comunicación. Destaca especialmente la Universidad Carlos III, que presenta un mayor conocimiento de la existencia de normas de seguridad y medidas sobre el correo electrónico. Por otro lado, observamos que la mayor parte de los docentes desconocen la existencia de controles (por parte de algunas empresas) de los contenidos del correo electrónico. Resulta llamativo comprobar que en la Universidad Rey Juan Carlos ningún docente conoce norma alguna sobre la utilización del correo, aunque una parte importante de ellos emplean medidas de seguridad.

Las tablas 11 y 12 muestran las frecuencias obtenidas de las ventajas e inconvenientes del correo electrónico destacados como más interesantes por los participantes

La principal ventaja percibida es la rapidez en la comunicación, que afirman casi 700 respuestas (recordemos que se obtuvieron 910). También son importantes las consideraciones de sencillez y facilidad en el uso, la posibilidad de enviar otros ficheros y el bajo coste. En cuanto a los problemas que se producen fruto del uso del correo electrónico, son los que se han destacado, por orden de prioridades, en la tabla 12 . 


\section{TABLA 11}

Ventajas del uso del correo electrónico

\begin{tabular}{|c|c|c|c|c|c|c|c|c|}
\hline & Ventajas & $U C 3 M$ & $U P M$ & $U C M$ & $U A M$ & $U A H$ & \multicolumn{2}{|c|}{ URJC TOTAL } \\
\hline 1. & Rapidez de información .................... & 129 & 134 & 184 & 91 & 120 & 40 & 698 \\
\hline 2. & Sencillez y facilidad $\ldots \ldots \ldots \ldots \ldots \ldots \ldots \ldots$ & 12 & 56 & 180 & 34 & 13 & 33 & 328 \\
\hline 3. & Transferencia de ficheros $. . . . . . . . \ldots \ldots \ldots . . . . .$. & 13 & 107 & 58 & 12 & 51 & & 241 \\
\hline 4. & 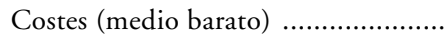 & 30 & 41 & 98 & 18 & 14 & 15 & 216 \\
\hline 5. & 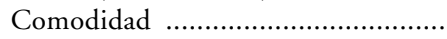 & & 13 & 30 & 13 & 54 & 15 & 125 \\
\hline 6. & 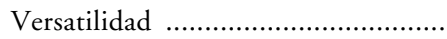 & 13 & 12 & 55 & 13 & 5 & & 98 \\
\hline 7. & Comunicación. & 4 & 36 & 14 & 13 & 15 & 12 & 94 \\
\hline 8. & 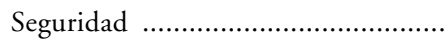 & 3 & 12 & 13 & 12 & 14 & & 54 \\
\hline 9. & Almacén de mensajes ....................... & 2 & & 12 & 1 & 8 & & 23 \\
\hline 10. & 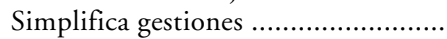 & 3 & 1 & & 13 & & & 17 \\
\hline
\end{tabular}

TABLA 12

Problemas en el uso del correo electrónico

\begin{tabular}{|c|c|c|c|c|c|c|c|}
\hline Problemas & $U C 3 M$ & $U P M$ & $U C M$ & $U A M$ & $U A H$ & \multicolumn{2}{|c|}{ URJC TOTAL } \\
\hline 1. Multitud de programas ...................... & 13 & 53 & 199 & 55 & 12 & 13 & 345 \\
\hline 2. Escasa intimidad o confidencialidad .. & 15 & 28 & 149 & 13 & 46 & 12 & 263 \\
\hline 3. Dedicación horaria alta ...................... & 36 & 36 & & 14 & & 34 & 120 \\
\hline 4. Hay mensajes que no llegan ............. & 13 & 2 & 36 & 35 & 17 & 13 & 116 \\
\hline 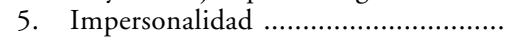 & 3 & & & 55 & & 3 & 61 \\
\hline 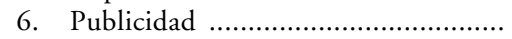 & 13 & 34 & 12 & & 1 & & 60 \\
\hline 7. Pérdida de información ..................... & & 36 & & 13 & 2 & & 51 \\
\hline 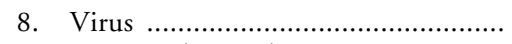 & & 35 & & & & & 35 \\
\hline 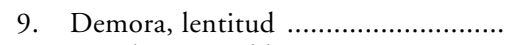 & & 13 & 3 & 1 & 2 & 14 & 33 \\
\hline 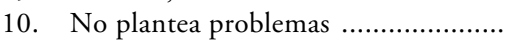 & 1 & 1 & 12 & 13 & 3 & & 30 \\
\hline 11. Falta de uso generalizado .................. & & 3 & 13 & 13 & & & 29 \\
\hline 12. Difícil acceso ................................... & 2 & & 12 & & 1 & & 15 \\
\hline 13. Fallos y problemas técnicos ................ & & 5 & & & & & 5 \\
\hline
\end{tabular}

El mayor inconveniente que encuentran los docentes en el uso de esta herramienta es la existencia de varios programas que gestionan el correo, lo que puede dificultar su comprensión y modificar ligeramente los formatos. Tampoco son escasas las respuestas que se refieren a la falta de confidencialidad o a la necesidad de importante dedicación temporal. Para remediar algunos de estos problemas, dos son las principales medidas de seguridad que han sido mencionadas en las respuestas: los antivirus y el uso de palabras claves.

La cantidad de ventajas percibidas por los usuarios es, en frecuencia, cercana al doble de los inconvenientes, de donde podríamos deducir que la satisfac- 
ción con el correo electrónico es alta. Los docentes lo consideran en general un medio ágil y barato de transmisión de información, que facilita los trámites y gestiones, con una importante versatilidad, aunque todavía no existe ningún estándar generalizado de correo $y$, en ocasiones, implica una pérdida de intimidad.

La posibilidad, e incluso legalidad, de un eventual control de los mensajes recibidos o enviados por correo electrónico es una cuestión controvertida. La siguiente tabla recoge las contestaciones a esta pregunta, clasificadas por universidades y agrupadas según sean favorables, desfavorables o intermedias.

\section{TABLA 13}

Opiniones sobre medidas de control en el correo electrónico

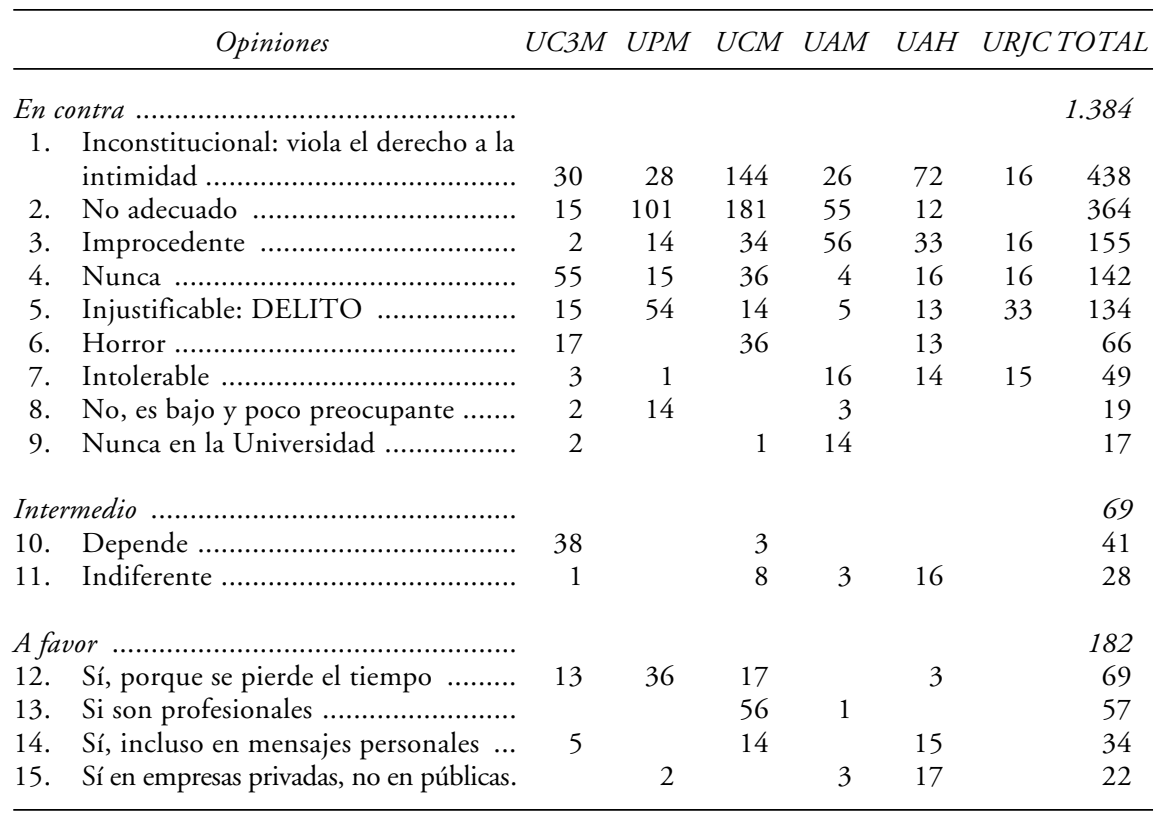

Lo primero que podemos deducir de las respuestas obtenidas es que la pregunta no ha dejado a mucha gente indiferente. La existencia de más contestaciones que cuestionarios recibidos implica que las opiniones expresadas son complejas, especialmente cuando son contrarias a este control, ya que los docentes apoyan su posición en diversos argumentos. Especialmente llamativa nos resulta la existencia de un colectivo que apoya el control de los mensajes cuando son profesionales, especialmente en un ámbito como el universitario, 
en que la confidencialidad de algunas investigaciones en curso resulta crucial para que éstas lleguen a buen fin.

La mayor parte de las opiniones favorables al control del correo electrónico se basan en la pérdida de tiempo que éste origina a algunos docentes, mientras que la mayor parte de posturas contrarias afirma que se trata de una violación del derecho a la intimidad, comparado por algunos de ellos con una escucha telefónica.

Como conclusión de este bloque, podemos afirmar que el correo electrónico es, por lo general, valorado de manera positiva y que, aunque no exento todavía de inconvenientes, los docentes lo consideran no tanto una herramienta con un gran futuro como el verdadero presente de la comunicación.

\subsection{ESTUdio de LAS CORRELACIONES}

A continuación procedemos al estudio de las correlaciones, con anterioridad a formular algunas de las hipótesis de trabajo.

Existe una correlación significativa (superior al 59\%) entre la actitud hacia los medios informáticos y la actitud hacia el correo electrónico. Parece lógica esta relación, dado que el medio empleado es el mismo (el ordenador), y el correo guarda fuertes relaciones con algunas de las herramientas informáticas (por ejemplo, procesadores de textos para enviar mensajes).

Ligeramente inferior, aunque también significativa, es la relación entre la actitud hacia el correo electrónico y el conocimiento del mismo, alineada con las teorías psicológicas de los medios informáticos. En la misma línea de razonamiento se encuentra la relación (cercana al 50\%) entre la actitud hacia el correo y su uso. Finalmente, y dentro del mismo bloque, notoriamente superior $(70 \%)$ es la relación entre el conocimiento del correo y su uso.

Para contrastar en cierto modo la veracidad de las afirmaciones anteriores, podemos observar que, aunque menor, existe una cierta correlación entre el conocimiento del correo electrónico y el tiempo de utilización del mismo (26\%). Por otra parte, el tiempo de utilización del correo también está relacionado con el número de mensajes enviados, aunque solamente al $16 \%$.

Las relaciones que antes hemos establecido con respecto al correo electrónico tienen también sentido aplicadas al resto de herramientas informáticas. Así, existen fuertes correlaciones (superiores al 75\%) entre el conocimiento y el uso de Internet, las bases de datos digitales y los paquetes estadísticos. Podemos razonar estas altas correlaciones, especialmente las dos últimas, dada la dificultad del aprendizaje de estas herramientas, que sólo se justifica si van a ser utilizadas.

El grado de conocimiento de Internet también se relaciona positivamente con el uso del correo electrónico (53\%). Además de la posibilidad de configurar el correo a través de Internet, esta relación se apoya en que ambas tecnologías pertenecen al entorno de la telemática. 
Dentro del campo de las curiosidades, debemos resaltar la correlación negativa fuerte (cerca del 54\%) que existe entre la edad de los encuestados y el porcentaje de mensajes que no tienen contenido laboral. La mayor parte de estos mensajes sin contenido laboral son enviados por los más jóvenes.

\subsection{FORMULACIÓN Y CONTRASTE DE HIPÓTESIS}

Teniendo en cuenta la Teoría de la Acción Razonada ${ }^{4}$ de Fishbein y Ajzenis, la actitud refleja la cantidad de afecto que uno siente "hacia o en contra" de algún objeto o comportamiento. La actitud de una persona en relación con la tecnología de la información se refiere a si la persona siente que la tecnología de la información es positiva o negativa. Davis et al. (1989) encuentran que la actitud de las personas hacia el uso de tecnologías de la información está relacionada directamente con la percepción que estas personas tienen de las tecnologías. Orlikowski y Gash (1994) argumentan que el conocimiento que las personas tienen sobre el comportamiento de las tecnologías es crítico para comprender su interacción con ella.

De estas teorías podemos derivar directamente las siguientes hipótesis:

H1: Existe una relación directa entre la actitud que las personas tienen hacia el correo electrónico y el conocimiento del mismo.

$\mathrm{H} 2$ : Existe una relación entre el conocimiento del correo electrónico y su utilización.

H3: Existe una relación directa entre la actitud hacia los medios informáticos y el conocimiento de los mismos.

H4: Existe una relación entre el conocimiento de los medios informáticos y su utilización.

Igualmente, podemos afirmar que el correo electrónico guarda una relación más estrecha con algunas herramientas informáticas que con otras. Así, y dado que la mayoría de los mensajes enviados actualmente tienen un contenido fundamentalmente textual, podríamos relacionar el conocimiento de los procesadores de textos con el uso del correo. Del mismo modo, por origen común y una cierta convergencia de tecnologías, postulamos una relación entre el conocimiento de Internet y la utilización del correo. Estas opiniones son apoyadas por el estudio de las correlaciones que hemos realizado con anterioridad.

H5: Existe una relación directa entre el conocimiento de los procesadores de textos y la utilización del correo electrónico.

H6: Existe una relación directa entre el conocimiento de Internet y la utilización del correo electrónico.

4 Theory of Reasoned Action. 
Al margen de estas hipótesis, derivadas directamente de la teoría y la observación, tendremos la ocasión de contrastar la existencia de algunas otras relaciones y condicionamientos entre variables.

El contraste de las anteriores hipótesis se ha realizado mediante el análisis de la varianza (ANOVA), pudiendo en todos los casos rechazarse claramente la hipótesis nula de igualdad de medias. De este modo, las relaciones entre actitud y conocimiento, por un lado, y entre conocimiento y uso, por otro, del correo electrónico, así como del resto de medios informáticos, quedan probadas. A continuación, indicamos los niveles de significación para el caso del correo.

TABLA 14

Valores de los análisis de la varianza

\begin{tabular}{|c|c|c|}
\hline Relación & F de Snedecor & $\begin{array}{c}\text { Nivel } \\
\text { de significación }\end{array}$ \\
\hline Actitud correo sobre Conocimiento correo ........................... & 40,23 & 0,000 \\
\hline 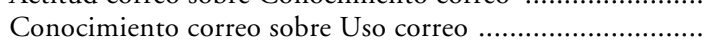 & 212,11 & 0,000 \\
\hline 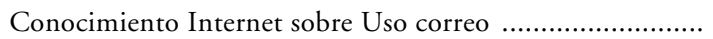 & 53,29 & 0,000 \\
\hline Conocimiento procesadores de textos sobre Uso correo ...... & 16,77 & 0,000 \\
\hline
\end{tabular}

Una aportación interesante, a la vez que sorprendente, es que, sin embargo, no se ha podido probar la existencia de una relación directa entre la actitud y el uso de los diversos medios informáticos, incluido el correo electrónico. Llegamos así a la conclusión de que la percepción inicial de un medio sólo se relaciona con su utilización real a través del aprendizaje del mismo, de los conocimientos que se adquieran.

El segundo bloque de hipótesis (5 y 6), relativas a relación entre diversos medios, también ha quedado probado, con lo que podemos afirmar que los medios informáticos que son percibidos como más relacionados con el correo electrónico son Internet y los procesadores de textos. Algunas de las características en común ya han sido indicadas, pero podríamos añadir otras, como son facilidad de aprendizaje, en general autodidáctico, o universalidad en las materias.

\subsection{TABLA DE CONTINGENCIAS}

Para finalizar, vamos a presentar una tabla de contingencias que recoge los valores de los usos y conocimientos para las diferentes áreas, de manera que quedan resaltadas las similitudes y las diferencias principales entre éstas. 


\section{TABLA 15}

Contingencias por área de conocimiento

\begin{tabular}{|c|c|c|c|c|c|}
\hline & $\begin{array}{l}\text { Ciencias } \\
\text { Sociales }\end{array}$ & $\begin{array}{l}\text { C. Tecnol. } \\
\text { Ingenierías }\end{array}$ & $\begin{array}{l}\text { Ciencias } \\
\text { Exper. }\end{array}$ & $\begin{array}{l}\text { Ciencias } \\
\text { Salud }\end{array}$ & $\begin{array}{l}\text { Humani- } \\
\text { dades }\end{array}$ \\
\hline 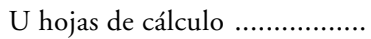 & Medio-bajo & Medio & Medio-bajo & Medio & Muy bajo \\
\hline U correo & Alto & Alto & Alto & Alto & Alto \\
\hline 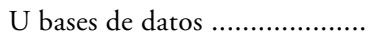 & Bajo & Bajo & Medio-bajo & Medio-bajo & Bajo \\
\hline 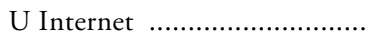 & Medio & Medio-alto & Medio & Medio & Medio \\
\hline U paquetes estadísticos ........... & Medio & Medio & Medio & Medio-bajo & Muy bajo \\
\hline U procesadores textos ............... & Alto & Alto & Alto & Alto & Alto \\
\hline $\mathrm{C}$ hojas de cálculo ...................... & Medio-bajo & Medio & Medio-bajo & Medio-bajo & Bajo \\
\hline 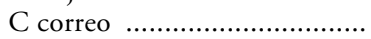 & Medio-alto & Alto & Alto & Alto & Medio-alto \\
\hline $\mathrm{C}$ bases de datos ................... & Bajo & Medio-bajo & Bajo & Bajo & Bajo \\
\hline 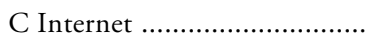 & Medio-alto & Alto & Medio & Medio & Medio \\
\hline C paquetes estadísticos ........... & Medio-bajo & Medio-bajo & Bajo & Bajo & Muy bajo \\
\hline $\mathrm{C}$ procesadores textos .............. & Alto & Medio-alto & Alto & Medio-alto & Alto \\
\hline Actitud medios ......................... & ++ & ++ & ++ & ++ & ++ \\
\hline Actitud correo .......................... & ++ & ++ & ++ & ++ & ++ \\
\hline
\end{tabular}

\section{CONCLUSIONES}

En este trabajo hemos tratado de realizar una breve aproximación a la utilización del correo electrónico en las universidades madrileñas. Para ello, hemos partido del conocimiento de una parte relevante de la teoría en torno a este medio de comunicación, y la hemos contrastado con los datos recogidos en la encuesta realizada.

El correo electrónico tiene en la actualidad una gran relevancia, y ésta es incluso mayor en el ámbito universitario. La necesidad de conocer datos e investigar conjuntamente con personas que se encuentran en otros centros mueve a los docentes a utilizar abundantemente el correo. Su alta versatilidad y su sencillez de manejo le convierten, potencialmente, en el medio de comunicación por excelencia para la faceta investigadora.

Existe una actitud muy positiva hacia el correo electrónico, que tiende a ser cada vez cada vez menos controvertida. Sólo algunos inconvenientes, como la ausencia de un estándar generalizado o la falta de confidencialidad, disuaden a algunos profesionales de su empleo mayoritario. Consideramos que su utilización aumentará con una mayor formación técnica de los usuarios (que conlleve mayor conocimiento del medio), y con la mejora de algunas incipientes y escasas herramientas de seguridad. Debemos, no obstante, resaltar la existencia de algunas diferencias significativas por universidades, áreas de conocimiento y categoría profesional, que consideramos que pueden tender a reducirse con el tiempo. 
Como futuras líneas de investigación, nos parece interesante una comparación de los resultados obtenidos en este trabajo con los que puedan resultar de estudios análogos realizados en las universidades privadas, entre el alumnado de universidades públicas y privadas o entre profesionales de otros organismos públicos.

Sin embargo, creemos que se podrían obtener resultados de mayor calado de una comparación entre los datos obtenidos en las universidades y los que pudiéramos observar en empresas radicadas en nuestro país, por considerar que algunas contestaciones presentarán diferencias presumiblemente significativas.

\section{BIBLIOGRAFÍA}

Anderson, R. H.; Bikson, T. K.; Law, S., y Mitchell, M. (1996): Universal access to e-mail feasibility and societal implications, Rand.

Brown, R. M.; Gatian, M. W., y HiCKS, J. O. (1995): «Strategic Information Systems and Financial Performance», Journal of Management Information Systems, vol. 11, n. ${ }^{\circ} 4$, primavera, pp. 215-248.

Clemons, E., y Row, M. (1991): «Sustaining IT Advantage: The Role of Structural Differences», Management Information Systems Quarterly, septiembre, pp. 275-292.

DAVIS, F. (1989): «Perceived Uselfulness, Perceived Ease of Use, and End User Acceptance of Information Technology", Management Information Systems Quarterly, septiembre, pp. 319339.

Dos Santos, B., y Peffers, K. (1993): «Firm Level Performance Effects: A Framework for Information Technology Evaluation Research», en Strategic Information Technology Management, Banker, Kauffman y Mahmood (eds.), Idea Group, cap. 23, pp. 515-546.

Erosa, V. E. (1996): «Exploring how Information Technology Affects Competitive Advantage in a Core Stable Technology Manufacturing Firm», Tesis Doctoral, Universidad de Texas at Austin-ITESM-CCM.

Eveland, J. D., y BiKson, T. K. (1988): «Work Group Structures and Computes Support: a Field Experiment", ACM Transactions on Office Information Systems, 4, 4, pp. 354-379.

Finholt, T., y Sproull, L. (1990): «Electronic Groups at Work», Organisations Science, 1, 1, pp. 41-64.

Fishbein, M., y Ajzen, Y. (1975): Belief, Attitude, Intentions and Behaviour: An Introduction to Theory and Research, Addison-Wesley, Boston (MA).

FULK, J. (1993): «Social Construction of Communication Technology», Academy of Management Journal, 36, 5, pp. 921-950.

GinZBerG, M. J. (1981): «Finding an Adequate Measure or OR/MS Effectiveness», Interfaces, vol. 8, n. ${ }^{\circ}$ 4, pp. 59-62.

IVES, B., y JaVEnPAA, S. L. (1991): "Aplications of Global Information Technology: Key Issues for Management", Management Information Systems Quarterly, vol. 15, n. ${ }^{\circ}$ 1, marzo, pp. 3349.

MarKUS, M. L. (1994): «Electronic Mail as the Medium of Managerial Choice», Organization Science, 5, 4, pp. 502-527.

MCFARlAN, D. (1984): «Information Technology changes the way you compete», Harvard Business Review, vol. 62, n. ${ }^{\circ}$ 3, pp. 98-103.

McKeen, J. D., y Smith, H. A. (1993): «The relationship between Information Technology Use and Organizational Performance», en Strategic Information Technology Management, Banker, Kauffman y Mahmood (eds.), Idea Group, cap. 20, pp. 405-444. 
OrLOWSKY, W. J., y GASH, D. C. (1994): «Technological Frames: Making Sense of Information Technology in Organizations», ACM Transactions on Information Systems, vol. 12, n. ${ }^{\circ} 2$, abril, pp. 174-207.

Porter, M. E. (1985): «Technology and Competitive Advantage», en Competitive Advantage: Creating and Sustaining Superior Performance, Free Press, N.Y., cap. 5.

Porter, M., y Millar, V. E. (1985): «How Information gives you Competitive Advantage», Harvard Business Review, julio-agosto, pp. 149-160.

RHEINGOLD, H. (1996): La comunidad virtual, Gedisa.

Rice, R. E., and Associates (eds.) (1984): The New Media: Communication, Research and Technology, Newbury Park (CA), Sage.

Ricoma, C. (1996): «Impacto de la Tecnología de la Información en la Comunicación Interna», Capital Humano, n. ${ }^{\circ}$ 90, junio, pp. 36-39.

MORTON, S. (1991): The Corporation of the 1990. Information technology and organizational transformation, Oxford University Press.

SeTHI, V., y KING, W. R. (1994): «Development of Measures to assess the extend to which an Information Technology Application provides Competitive Advantage», Management Science, vol. 40, n. ${ }^{\circ}$ 12, diciembre, pp. 1601-1620.

SILVER, M.; LYNNE, M., y MATHIS, C. (1994): «The information technology interaction model», Work Paper series, STERN IS 94-5, Stern School of Business, Center for Research on Information Systems, Nueva York University, obtenido en Internet en http://www.nyu.edu.

Sproull, L., y Kiesler, S. (1991): Connections: New Ways of working in the Networked Organization, Cambridge (MA), The MIT Press.

TORASCAR, K., y JOGLEKAR, P. (1993): «Applying cost-benefit analysis methodology for Information Technology Investment Decisions", en Strategic Information Technology Management, Banker, Kauffman y Mahmood (eds.), Idea Group, cap. 6, pp. 119-142.

Treviño, L. K.; Lengel, R. K., y DAFt, R. L. (1987): «Media Symbolism, Media Richness and Media Choice in Organizations», Communication Research, 14, 5, pp. 553-574.

Weill, P. (1992): «The Relationship Between investment in Information Technology and Firm Performance: A Study of the Valve Manufacturing Sector», Information Systems Research, vol. 3, n. ${ }^{4}$, diciembre, pp. 307-333.

- (1993): "The Role and Value of Information Technology Infraestructure: Some Empirical Observations», en Strategic Information Technology Management, Banker, Kauffman y Mahmood (eds.), Idea Group, cap. 24, pp. 547-572.

WiLSON, D. (1993): «Assesing the Impact of Information Technology on Organizational Performance», en Strategic Information Technology Management, Banker, Kauffman y Mahmood (eds.), Idea Group, cap. 22, pp. 471-514.

YATES, J., y ORLIKOWSKI, W. J. (1992): «Genres of Organizational Communication: A Structurational Approach to Studying Communication and Media», The Academy of Management Review, 17, 2, pp. 299-326.

- (1994): «Genre repertorie: Examining the Structuring of Communicative Practices in Organizations», Administrative Science Quaterly, 39, 4, pp. 541-574.

YIN, R. K. (1994): Case Study Research Design and Methods, Sage. 


\section{ABSTRACT}

Electronic mail is one of the communication methods that promises to be essential in communications in the next few years. The university world cannot remain outside this reality for reasons of its very characteristics, in terms of teaching and research alike.

With a view to verifying the real introduction of electronic mail into the public universities in Madrid, we have designed and carried out a survey which aims at collecting details of the opinion held by teachers concerning its use and a knowledge of electronic mail and other computer media, and their personal attitude towards them. We have also taken an interest in some matters of relevance concerning electronic mail, such as the users' level of satisfaction and the expectations this method of communication raises.

An empirical comparison has been made between the existence of different relationships, placing an emphasis on the influence of prior attitudes in the levels of knowledge of the tools, and the influence of such knowledge on their subsequent use. Similarly, we have been able to demonstrate the existence of significant differences between the different areas of knowledge, professional categories and universities. 


\section{TEXTO CLÁSICO}

